\title{
An Analysis of Stability of Inter-bank Loan Network: A Simulated Network Approach
}

Saurav Chakraborty

U of South Florida

$\underline{\text { sauravc@mail.usf.edu }}$
Jordan Gaeta

IU Bloomington

jvgaeta@indiana.edu

\author{
Kaushik Dutta \\ U of South Florida \\ duttak@usf.edu
}

\author{
Donald Berndt \\ U of South Florida \\ dberndt@usf.edu
}

\begin{abstract}
Research in the domain of Financial Contagion has come to the forefront in recent years. There has been a significant focus on this field since the recession of 2008. In this paper we take a look at simulation based modelling to stress test the stability of inter-bank loan networks of different structures. We look to analyze the effect of various parameters on the stability of these networks. We first simulate networks which are Homogeneous in nature. We then simulate a Heterogeneous (tiered) network. The model also introduces an endogenous loaning mechanism to imitate a more realistic inter bank loan market. We run simulations on these networks to gain a better understanding of the propagation of losses through the network. After studying the results of these simulations we come up with some interesting new insights about how parameters like connectivity and size of the network, effect a tiered intra-bank financial network. One of our key findings is that higher inter-tier connectivity is good for the stability of big banks but not so much for banks of smaller size.
\end{abstract}

\section{Introduction}

The recent global recession of 2008 was a global disaster. It led to losses in the home equity network that transpired to huge losses being incurred in the stock network. According to Business Insider, the United States of America lost more than 10 Trillion dollars in the crash [19]. According to The Guardian, the crash in network also led to more than 500 banks declaring bankruptcy and a considerably higher number of banks suffered considerable losses, in the subsequent years [20]. Modern day financial institutions are interlinked between each other. The failure of one institution affects the financial health of others. This is depicted by common "Too Big to Fail" phrase [21] which indicates there are certain big financial institutions that can't be allowed to fail. The failure of these banks will lead to catastrophic failure of the overall financial systems. However, though there is an agreement that there are few banks that are too large and too risky to fail, there has not been much research or study on how big is too big. There is scant understanding of the impact of various characteristics

of financial networks that may affect the stability of the network. In light of this tragedy, the study of systemic risk in financial Networks has come to the forefront of research. Acemoglu et al [3] have studied this aspect through an analytical way. They have mathematically modeled the financial network and have identified several properties and characteristics for stability of the financial network.

Though mathematical modeling is important for deriving theoretical insights, it is limited by the assumptions that are needed to make a mathematical model fit to a real life scenario. To address more realistic situation, in this paper we use simulation based network modeling to identify scenarios where failure of one or few banks can lead to failure of the overall system. Through simulation based approach we determine, how Failure of one individual bank in a financial network can cause a cascade of failures throughout the system. We take a network model approach [1] [2] [3] [4] [5] to do this simulation. In this research we focus on the financial sector, specifically interbank loan networks.

First we simulate the interbank loan network through a simulation of a network of nodes. Next, to do stress test of the network, we simulate shocks which can assess the stability of the network based on certain parameters. A concept known as contagion is introduced which affects the whole network of nodes, instead of just one bank. The banks have dependencies on the other banks when they either borrow money or lend money to other banks. Such cases are also handled in this model of banking. Let us consider an example of a bank which goes bankrupt and crossed the limits of capital. The loans it has got have exceeded the limits and it cannot repay the loan amount now. In such 
cases, the lending banks would be losing the money and their net capital (assets) should be updated accordingly. If a bank's asset becomes lower than its liabilities, we say it has defaulted and remove it from the network. However, removal of one bank from the financial system leads to loss of the loan given to the removed bank by all other banks in the system. This leads to propagation of financial loss through the network and may lead to failure (or removal) of more banks from the system. As the number of banks failing due to such a domino effect increases, more unstable the financial network becomes.

In this research we investigate the stability of this interbank loan network against certain parameters number of banks, their internal connectivity and the severity of a shock. We measure the stability by the metric of how many nodes and how much residual capital survives in the aftermath of the shock. We also analyze a tiered banking network structure with an endogenous loaning mechanism, where the decision of granting an interbank loan is not random but is based on risk and return of the loan.

This research is fundamental to the kind of research being sought after by Central Banks around the world [11][12]. At the junction of financial crisis, the output of this research can be used by central banks to determine when and how to intervene. For example, whether the central bank should let a bank fail or should it intervene - this question has wide political and economic implications. However, seldom that decision is driven by evidence based research. This research aims to close that particular gap.

Some of the specific contributions of this paper are to run simulations on tiered networks resembling the real world inter bank loan markets and see how connectivity and size of the network along with the intensity of the shocking mechanism effect the stability of such a tiered structure. We analyze in detail the effect of such parameters on each individual tier of the said banking network.

The rest of the paper is organized as follows in section 2 we discuss the Related Works to this kind of research. In section 3 we describe in detail the model and define the key terminologies that have been used in the paper. Here we also describe the various methods used in the model like the shocking mechanism, the endogenous loaning mechanism and how losses propagate in the system. In section 4 , we discuss about the metrics used and analyze the results achieved after running simulations. This is followed by Conclusions.

\section{Related Work}

The way we look at the problem is very similar to the way a disease spreads through a population where the infection can be transmitted from one person to another upon contact [13] [14]. This is where the name contagion is derived from. This approach of determining the stability of a network or propagation of a shock has been studied in various other disciplines like Biology [13] [14], Economics [15] [17], Psychology [16] and Sociology [18] among many others.

Even though the interest in contagion has seen significant spike in recent years due to the global crisis, it is not a new topic, Diamond and Dybvig [22] came up with a model for bank runs and related financial crises way back in 1983. There was also considerable research done in the field after the Mexican Peso crisis of 1994 and the Asian Network crash of 1997 [23][24]. However the domain really came to the forefront with the influential paper by Allen and Gale [17] where they analyzed the fragility of a given network system based on its structure by making use of simple examples. There has been a lot of theoretical research done in the study of contagion [3] [4] [6] [7] [8] [9] [10], but there is a lack of empirical research of the field. This lack of empirical research has been a problem for central banks and regulators. Many central banks around the world have conducted research that looks to address this issue [11] [12] [8]. However as the data required for this kind of research is proprietary in nature, research outside of institutions with available data, need to resort to simulations. This has led to the use of simulations to study the effect stability of network of financial institutions in case of instability of one or few banks in the network.

Nier et al [5] were one of the first to use simulation methods to analyze the interbank loan networks. This work, in turn, has greatly inspired a lot of further research in this domain. The use of Eboli model which equates the default dynamics of the interbank loan networks to flow networks in physics, led to this work being emulated and improved on further by multiple papers. One such paper being Dasgupta et al [1] who use simulations to come up with a contagion index combining various characteristics of similar network models as of [5]. Upper [2] in 2011 used simulations to validate the results and compare the various models used by different papers; this paper showcases how simulations can be used to visualize the loan networks as networks or graphs and how it can be a powerful tool for analyzing interbank loan networks.

However, most of these simulations have been run on Homogeneous networks, networks where all nodes and 
edges are of equal importance/strength. There has been few studies with a two tiered network structure [1] [2] [5] [6] [7] [9], where nodes are identical to nodes in its own tier but differ from nodes of the other layer. Also the edge weights vary leading to this structure being very different from the Homogeneous one. Many have argued that even though the results achieved in a homogeneous network may work in an ideal world, the complexity of the real world problems can be represented by adding Heterogeneity to the said networks. So, [1] and [5] delve into heterogeneity by running simulations on a two tiered alpha-beta heterogeneous network, [7] investigates the existence of dense cores in a heterogeneous network of banks. In this paper we extend this work by running simulations on a three tiered structure, with heterogeneity among nodes in each layer. We emulate a real work bank lending network by introducing a third tier which shall resemble the sector specific financial institutions (such as mutual fund or other financial derivatives of various sectors). Here the tier 1 bank is the 'Big' banks, so they will have a considerably large amount of assets whereas second tier banks will be relatively smaller in size. The tier 3 will refer to sectors and they represent a collection of loanees pertaining to specific domains. So for example Real Estate can be regarded as a sector. None of the banks will be identical to each other. We first validate the results achieved for Homogeneous structures and compare them to results obtained by [1] [2] and [5]. We then show that similar relationships can be observed for a more complex three tiered structure as well and derive few more useful insights.

\section{Model and Method}

In this section we describe the model and the approach in simulating the interbank loan networks. We represent each entity in the interbank loan networks (such as a bank) as a node in a directed graph. So $\mathrm{G}=$ $(\mathrm{V}, \mathrm{E})$ is the network representation of interbank loan network. $\mathrm{V}$ is the set of all nodes in the network, where each node represents a financial institution (banks) that takes part in the interbank loan. An edge $\left(v_{1}, v_{2}\right) \in E_{v} \forall v_{1} \in V$ and $v_{2} \in V$ is a directional edge from $v_{1}$ to $v_{2}$ represents a loan given by node $v_{1}$ to node $v_{2}$. Each node has one important characteristic, weight (called node weight) which represents the cash in hand with that node (financial institution). Each edge has also a weight, which represent the current loan amount from node $v_{1}$ to $v_{2}$.

To model the interbank loan network we first assume the interbank loan networks as a network of nodes and edges. We allocate node weights to all the nodes and edge weights to all the edges, once that is done we sum up all the node weights to get the total net capital of the network and denote it by N. Similarly we sum up all the edge weights to get the total lending or borrowing in the network and denote it by $\mathrm{E}$. The ratio of these two play an important role as we will see later and we denote it as the N/E ratio.

Before describing the details of the loan network, below we first describe few concepts.

Systematic Risk: Systemic risk led to financial crisis in 2008. It is a problem in banking, where an isolated event triggers instability or collapse of the whole system. In a banking network, due to a lot of dependencies, if one of the banks fail, there may be a chance of cascading failure, so losses can propagate to all the other banks in the chain. Systemic risk is a major concern for central banks, in safeguarding the whole financial stability. It occurs, if some of the banks in the network have high potential to fail and indirectly impose the same onto the whole economy. We check for systemic risk in our simulated network by providing shock to the system and removing banks which default.

Each node in the simulated interbank loan financial network is a bank or financial entity that can borrow or lend money in the lending network. Below we define some common financial terminologies that are used to denote the health of a bank. These characteristics will be associated with each node in our model and will be used to determine the health of the node.

Balance sheets, Assets, Liabilities, Capital: Balance sheet is a report which shows the bank's assets, liabilities and the equity share of all the owners [25]. An asset has a value which can be converted to cash by selling those [25].

Liability is an obligation that must eventually be paid as it is a claim on assets [25]. The owner's equity in a bank is often referred to as bank capital [25]. We can quote these as an equation [25],

Assets $=$ Liabilities + Capital

Shock: Now in this model similar to [1] [5] we introduce an external shocking mechanism. This mechanism has two important characteristics. First, across how many banks, such shock is introduced and second, if a bank is effected how much of its capital does it lose. The second parameter can be regarded as the severity of the shock, denoted by $\varphi$, whereas the first feature is deemed as the reach of the shock, denoted by $\chi$. If a bank is hit by a shock, we calculate its remaining capital by the following equation: 
Remaining capital $=(1-\varphi) *($ Assets $)$

The remaining capital definition comes in handy to ascertain when a bank fails in our model. Given an external shock or a hit we will deem a bank to be bankrupt or fail when its remaining capital becomes less than its liabilities. Once a bank fails, we remove it from the network, and the loans given to that failed bank from other banks are deducted from their respective assets. So this causes them to lose money and thus the initial shock causes losses to propagate throughout the entire network. This continues iteratively till there is no more bank in the system whose remaining capital is less than its liabilities. The interbank loan network can follow two different structures which we are going to discuss next.

\section{Homogeneous Inter Bank Loan Network}

In this kind of network each node (bank) is identical to all the other nodes in the system and each edge is identical to all the other edges in the Network. So, all the node weights will be equal to each other and all edge weights will be equal to each other. These types of networks represent those scenarios where all commodities in the system are equal to each other, they are assumed to be of the nature of Erdos Renyi graphs [1]. Though the homogenous assumption is not realistic, due to reduced complexities most existing research [1] [5] [7] on interbank loan network assumes homogeneous network. Such assumptions make the model analytically trackable. To make our research continuation from the past research, we first study the simulation based analysis on homogenous network to establish the results based on existing research.

In this structure all banks will have the same net capital, so if the total net capital of the network is denoted as $N$ and there are $n$ banks, each bank thus, will have a capital of $N / n$. Similarly if the total value of the loan network (the amount that has been given loan by one bank to another bank) is given as $E$ and there are $m$ interbank loans (i.e. edges), each edge in the homogeneous network will have an edge weight of $E / m$.

In our simulation of homogenous interbank loan network, a $\chi$ fraction of banks will be hit by a shock, where each of the banks will lose $\phi$ fraction of their assets. If the remaining capital of any bank is less than its liabilities, we will deem it to be bankrupt. After the initial shock we will see how many of the banks default and we will observe how much loss they propagate to banks who had lent them money. So this phenomenon takes in the form of a contagion and we observe how losses propagate throughout the network causing knock-on defaults. The number of surviving nodes after the network has stabilized is deemed to be the stability factor, and this is what we analyze as a measure while running our simulations. We will discuss the results in the next section. Next, we introduce more complex Heterogeneous interbank loan networks.

\section{Heterogeneous Inter Bank Loan Network}

To model the more realistic situation where both banks and loans are of varied size, we resort to heterogeneous network. Here, each edge and node in the network has individual values, which are unequal. As such heterogeneous networks are difficult to track analytically, existing research in this domain has not dealt with this kind of network in great detail. Even in existing research, where heterogeneous networks have been discussed [1][5], they assume $\alpha-\beta$ heterogeneity. $\alpha-\beta$ heterogeneity refers to the structure where $\alpha$ fraction of banks have $\beta$ fraction of the assets, generally for more heterogeneity $\alpha$ is close to 0 , like 0.05 and $\beta$ is closer to 1 , say 0.9 . In this research we will not make any such assumptions about the loan network. Here, with the help of simulation we plan to analyze the stability of an endogenous heterogeneous interbank loan network - which is the key contribution of this paper.

In our model we will not have the $\alpha-\beta$ heterogeneity. We will simply allocate Tier1 banks to be big banks with a very high amount of assets (e.g. JP Morgan Chase) and similarly the Tier 2 banks (e.g. any community bank) to have a considerably lower amount of assets. Tier 1 and tier 2 banks invest money in the third tier which are the various sector specific financial institutions to make money, as depicted by the figure below. We allow tier 2 banks to take loans from tier 1 banks so that they can have more capital to invest in the network sectors. Unlike in homogeneous structure, here we have a rate of return associated with each loan. For each dollar a tier 1 bank loans to a tier 2 bank, it has to return $r$ dollars, where $r>1$, the exact value of $r$ varies from loan to loan (i.e. for different edges). Similarly the loan to a sector has a rate of return $R$ which vary from loan to loan, but we always have $R>$ $r>1$, for all $R$ and $r$. Also there is a time paradigm in our model, it is built in a way that each loan from tier 1 to tier 2 needs to be repaid in 2 time stamps, whereas each loan to tier 3 gets repaid within the next time stamp itself. This is done so that the lower level banks can maximize each loan. Also, there is a risk of failure associated with investing in a sector. Typically as the rate of return of loan in a sector increases the risk of failure of returning on the loan increases too. 
These loans and investments are not done randomly but there is an endogenous decision making involved. Suppose a tier 1 bank does not wish to invest in a certain sector due to past losses, then it will not loan to tier 2 banks which invest money in that particular sector. Also a tier 2 bank which is financially sound will seek to invest in sectors which have a lower risk of failure, whereas a bank which is struggling financially, may choose to take greater risks to make sure they do not go under and is able to repay all the money it has borrowed. So each loan granted or each investment made during the simulations is not completely random in nature but follows these realistic constraints.

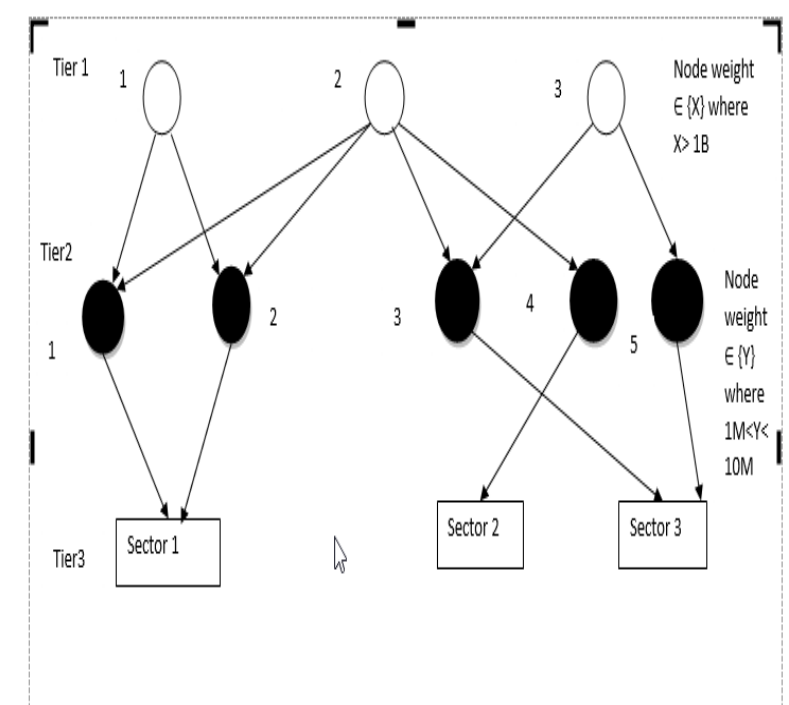

Figure 1 A sample 3-tiered Heterogeneous Network

In simulating heterogeneous network, we introduce the external shock similar to homogeneous network, but instead of hitting banks with shocks, here the sectors are targeted. If a sector is hit by a shock, all banks who had invested money in that particular sector, lose the money invested. Once the initial shock is setup we iteratively check if any of the banks default or not, if so how many of the banks go bankrupt and then analyze how much losses they propagate to banks who had lent these money.

In our heterogeneous network simulations, we observe the number of surviving nodes in each tier along with the total amount of money left in the system. We do this because we believe the amount of the money left in the network is a better representative of the state of the network. So we run our simulations to check both the number of surviving nodes as well the leftover amount of money to analyze our results.

\section{Results and Discussions}

In this section we describe the results obtained by our simulated experiments.

\section{Metrics Used}

To describe the current condition of the system, we focus primarily on two metrics.

Number of surviving nodes: This is the metric which has been chosen repeatedly in the past as well [1] [2] [5]. The objective of this study is to analyze the stability of the network. It is a good measure to see how many of the initial nodes, survived the contagion. We reported this result for both the homogenous and heterogeneous networks.

Percentage of money left in the system: In heterogeneous network where the asset of each bank is not equal, this is an important metric. For example, if 30 out of 100 banks survive the contagion, but in total they have only 30 dollars left as asset - that's not a good indication of the stability of the network. So we use the percentage amount left in the system (computed as sum of assets left over for all the surviving nodes) compared to the total amount in the system before the shock as a metric for stability of the network.

\section{Results}

The simulations we run, generate plots which help us interpret how each parameter affects the stability of the network. Each simulation run, we do, will vary a selected parameter, over a range of values as given in Table 1 and Table 2, while keeping all others constant at the benchmark values. The values that have been chosen as parameter ranges are based on past research done in this domain. They are inspired from the work done by Dasgupta et al. [1], Nier et al.[5] and Upper[2]. For each result we run a 100 simulations and then average our findings. These experiments will help us to understand how the parameters being examined influences the stability of the network and whether or not should be a factor to be monitored while investigating systemic risk.

Another interesting aspect is the ratio of the total asset with the total money being loaned/borrowed in the network. This is denoted by N/E ratio and is computed as sum of the node weights to the sum of the edge weights. We observe the effect of N/E ratio across all the simulations we run. For each of these parameters that we are examining we plot multiple times with different values of the N/E ratio.

\section{Homogeneous Network}

Here in each experiment we introduce shock with $\chi$ reach and $\varphi$ severity. We observe how that translates into banks getting bankrupt either with directly being 
shocked or indirectly through losses propagating from bankrupt banks in the network. This continues until the network becomes stable, that is there are no more banks going bankrupt. The number of surviving banks after the network stabilizes is what we are measuring to denote the stability of the network.

Effect of Connectivity: In this experiment we look at the effect of connectivity over the stability of the network. So here we vary the probability of forming a link (i.e. a bank has provided loan to another bank in the network) between two nodes from 0.05 to 0.95 , while keeping all other parameters at the benchmark value.

In Figure 2, we plot the number of solvent banks (nodes) as the edge probability increases. From Figure 2 , we see that with increased connectivity, number of surviving nodes after the shock stabilizes increases, making the network more stable. This result demonstrates that for a network, higher connectivity is better. Banks, the more they are connected with other banks, tend to be more stable as any loss coming in is shared by a number of banks and helps in reducing the effect of the shock.
Conclusion 1: Higher connectivity in the network in an interbank loan network, increases the stability of the network. Thus banks should loan to multiple other banks to increase their stability.

Also the effect of N/E ratio is also quite clear. With a high N/E ratio, the network is more stable even at low values of connectivity.

Conclusion 2: Banks should keep higher N/E ratio. Thus the banks should be careful about how much money they lend compared to the total assets they have.

The first conclusion encourages the diversification, which is quite well known in financial market. The second asks the bank to increase the reserve they have. After the 2008 financial crisis the banks have increased their reserve [26]. The above conclusion and simulated demonstration support that action.

Table 1: Simulation Parameters for Homogeneous Network

\begin{tabular}{|c|c|c|c|c|}
\hline $\begin{array}{l}\text { Name of the } \\
\text { parameter(Variable) }\end{array}$ & Description & Values / Range & $\begin{array}{l}\text { Benchmark } \\
\text { value }\end{array}$ & $\begin{array}{l}\text { Value } \\
\text { Distribution }\end{array}$ \\
\hline Node total & Total assets in the network & $400 \mathrm{M}, 800 \mathrm{M}, 1200 \mathrm{M}$ & & \\
\hline Edge total & Total amount of loans in the network & $800 \mathrm{M}$ & & \\
\hline Node weight & Asset of individual bank & $(10 \mathrm{M}, 40 \mathrm{M})$ & & Uniform \\
\hline Edge weight & $\begin{array}{l}\text { Amount of the total loan from one bank (origin node) } \\
\text { to another bank (destination node) }\end{array}$ & $(1 \mathrm{M}, 15 \mathrm{M})$ & & Uniform \\
\hline $\mathrm{N} / \mathrm{E}$ ratio & The ratio of the Node total to the Edge total. & $0.5,1.0,1.5$ & 1.0 & \\
\hline Number of nodes & Number of banks/financial institutions & {$[10,100]$} & 30 & $\begin{array}{l}\text { Uniform } \\
\text { increments of } 5\end{array}$ \\
\hline $\begin{array}{l}\text { Connectivity of the } \\
\text { network }\end{array}$ & $\begin{array}{l}\text { Probability of forming a directed link between any two } \\
\text { nodes (i.e. probability that a loan exists from one bank } \\
\text { to another bank) }\end{array}$ & {$[0.05,0.95]$} & 0.5 & $\begin{array}{l}\text { Uniform } \\
\text { increments of } \\
0.05\end{array}$ \\
\hline$\Phi$ & $\begin{array}{l}\text { Severity of the shock } \\
\text { (percentage of a bank's asset wiped out by the shock) }\end{array}$ & {$[0.05,0.95]$} & 0.95 & $\begin{array}{l}\text { Uniform } \\
\text { increments of } \\
0.05\end{array}$ \\
\hline $\mathrm{X}$ & Reach of the shock (\% of banks affected by shock) & {$[0.5,1]$} & & Uniform \\
\hline
\end{tabular}

Table 2: Simulation Parameters for Heterogeneous Network

\begin{tabular}{|l|l|l|l|l|}
\hline $\begin{array}{l}\text { Name of the } \\
\text { parameter(Variable) }\end{array}$ & Description & Values / Range & Benchmark & Distribution \\
\hline $\mathrm{n}_{1}$ & Number of banks in tier1 & $8,30,50$ & 30 & \\
\hline $\mathrm{n}_{2}$ & Number of banks in tier2 & $10,50,80$ & 50 & \\
\hline $\mathrm{n}_{3}$ & Number of nodes in tier 3 & $4,6,15$ & 6 & \\
\hline Asset Range1 & Range of Assets of Tier 1 & {$[10000,14000]$} & & Uniform \\
\hline Asset Range2 & Range of Assets of Tier 2 & {$[1000,8000]$} & & Uniform \\
\hline Loan Range 1 & $\begin{array}{l}\text { Range of total loans from } \\
\text { tier1 to tier 2 }\end{array}$ & {$[10000,13000]$} & & Uniform \\
\hline Loan Range2 & $\begin{array}{l}\text { Range of total loans from } \\
\text { tier 2 to sectors }\end{array}$ & {$[6000,8000]$} & & Uniform \\
\hline $\mathrm{X}$ & $\begin{array}{l}\text { Fraction of sectors } \\
\text { shocked }\end{array}$ & $(0.05,0.95)$ & 0.5 & Increments of 0.05 \\
\hline
\end{tabular}




\begin{tabular}{|c|c|c|c|c|}
\hline Inter-Tier connectivity & $\begin{array}{l}\text { Number of Tier } 2 \text { banks a } \\
T_{1} \text { bank is connected to }\end{array}$ & $5,25,50$ & 6 & \\
\hline Rate of return & $\begin{array}{l}\text { Return for each dollar you } \\
\text { invest in a sector }\end{array}$ & $(1.05 \rightarrow 2.0)$ & & Uniform \\
\hline Risk of failure & $\begin{array}{l}\text { This is the probability, that } \\
\text { a sector will fail and the } \\
\text { money invested in it will } \\
\text { be lost }\end{array}$ & $(0.05 \rightarrow 0.8)$ & & $\begin{array}{l}\text { Uniform (higher rate of } \\
\text { return is associated with } \\
\text { higher risk of failure) }\end{array}$ \\
\hline
\end{tabular}

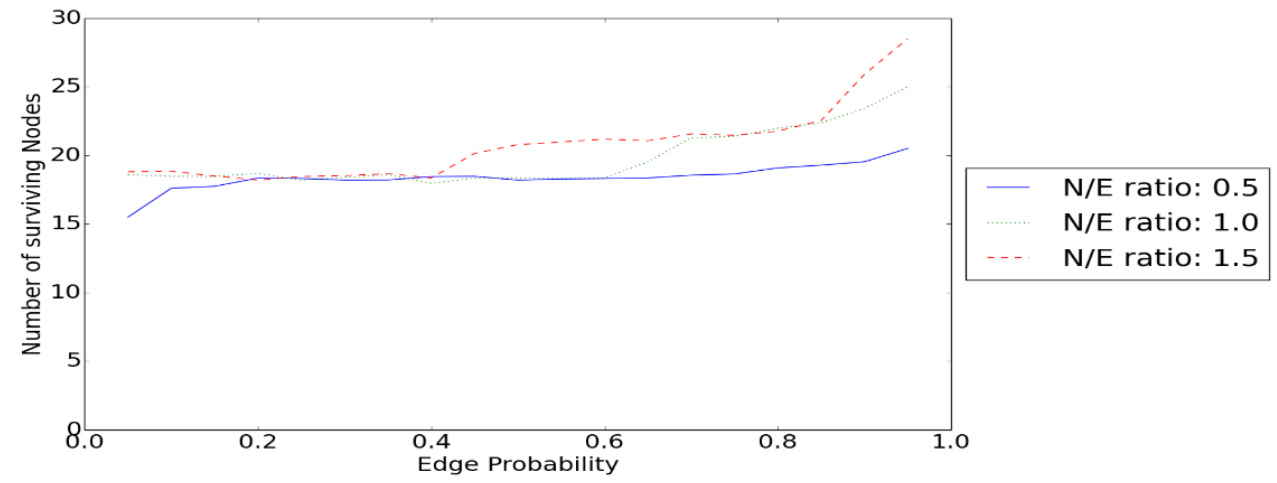

Figure 2: Effect of Network Connectivity in homogenous network

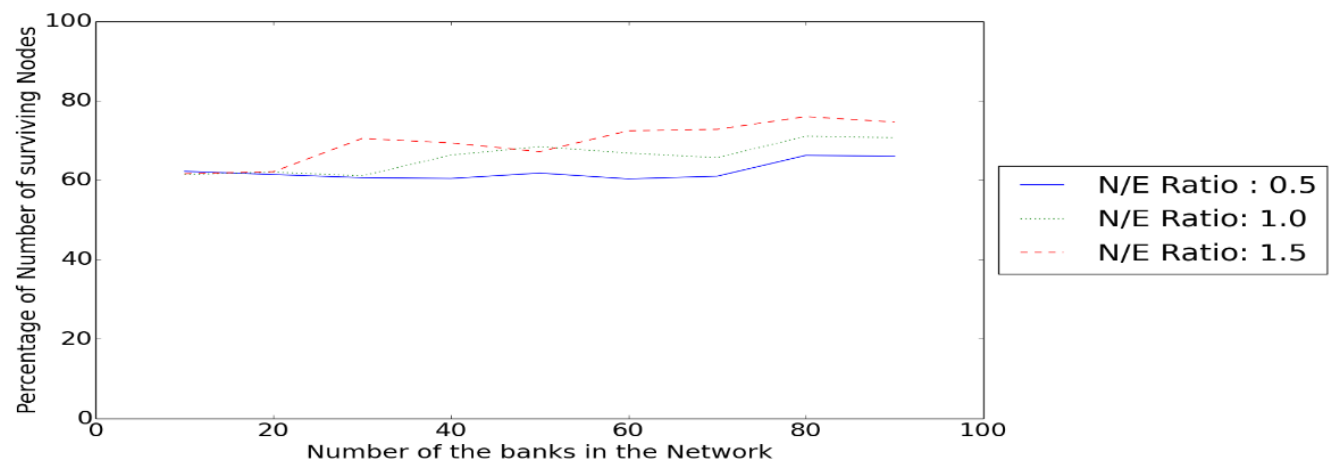

Figure 3: Effect of Number of banks in Homogenous network

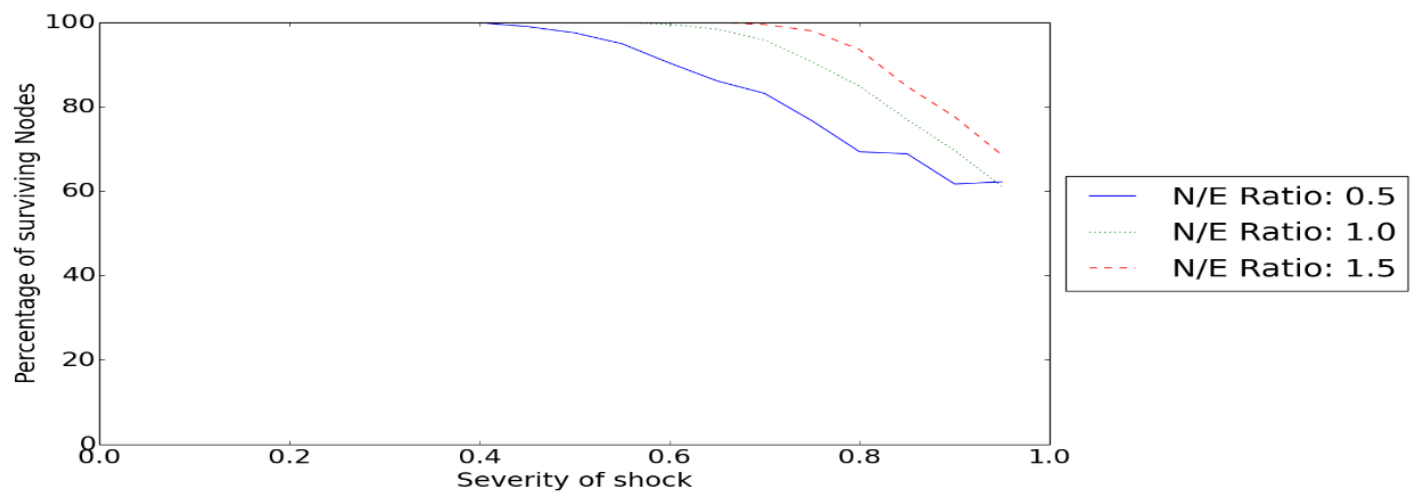

Figure 4: Effect of severity of shock in Homogeneous network

In Figure 3, we look at the effect that number of banks has on its stability. Here we see that with increased size (i.e. increased number of banks in the network), the financial loan market network becomes more stable.
We also observe that with higher N/E ratio more nodes survive the shock thus making the network more stable, this supports conclusion 2 above. 
Conclusion 3: More banks should be created and made part of the overall loan network. With increased number of financial institutions the stability of the overall network increases as depicted by the higher number percentage of surviving nodes after the shock. In Figure 4, we investigate the stability of the network as the severity of shock is increased. Figure 4 plots percentage of surviving nodes as the severity of the shock increases. We observe though at low severity of shock the stability of the network remains (i.e.

percentage of surviving node) same, at higher severity the stability of the network decreases drastically. We also note that as the N/E ratio increases, the decrement in the percentage of surviving node starts at much higher severity of shock, indicating higher stability of the network with higher N/E value.
Conclusion 4: Higher severity of shock leads to much severe damage to banking network.

The simulation on homogenous network led us to known facts - which validated our approach. Next, we describe the simulation of heterogeneous network which revealed some interesting new insights.

\section{Heterogeneous Networks}

In heterogeneous network simulations, we determine the stability of the network at various severity of the shock as the size of the network is varied and as the connectivity of the network is varied.

Each node in the heterogeneous network has different asset amount. The loan amount between various banks is also different. So in heterogeneous network we use the residual value of the total capital (as percentage of the initial total capital in the network) as the metric for network stability in heterogeneous network.

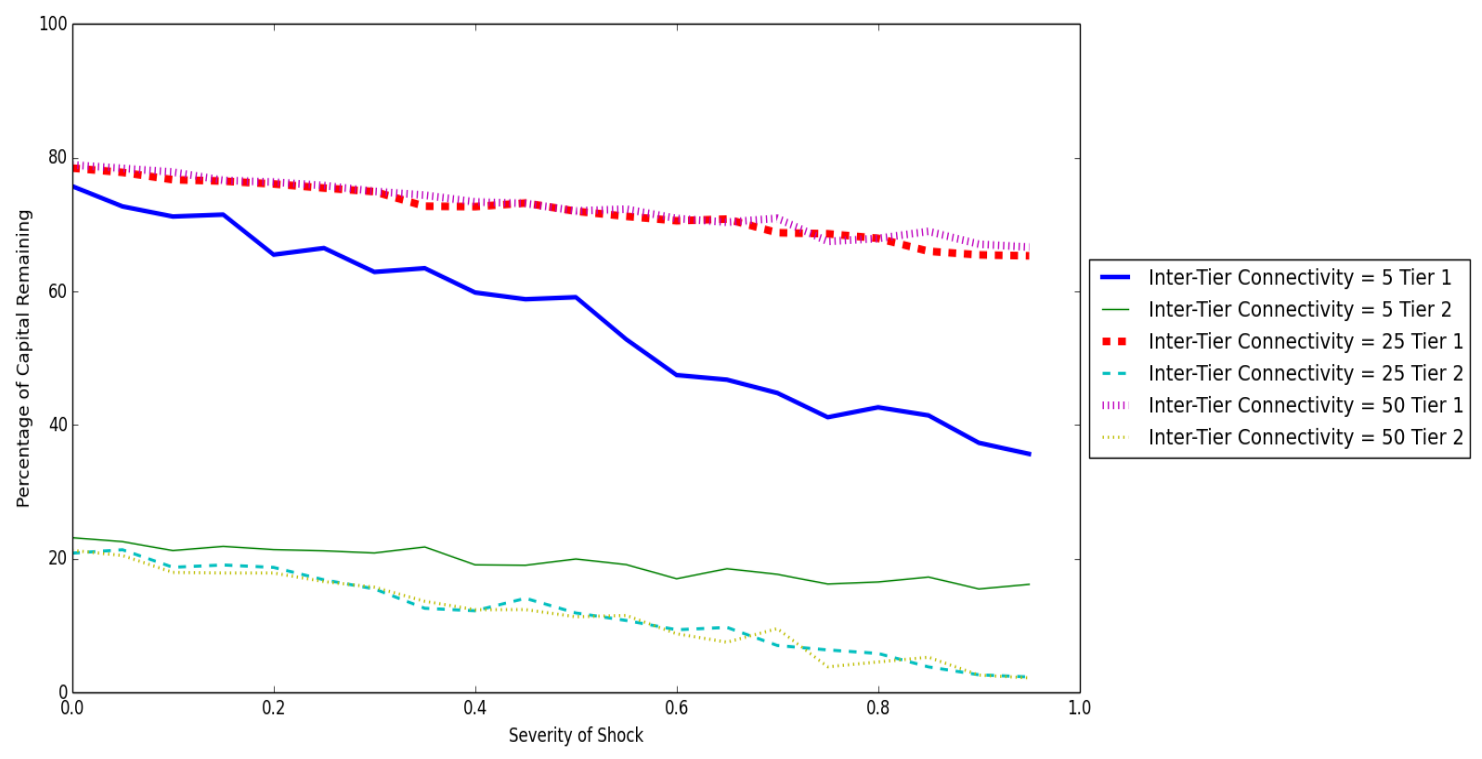

Figure 5: Effect of connectivity in a Heterogeneous Network 


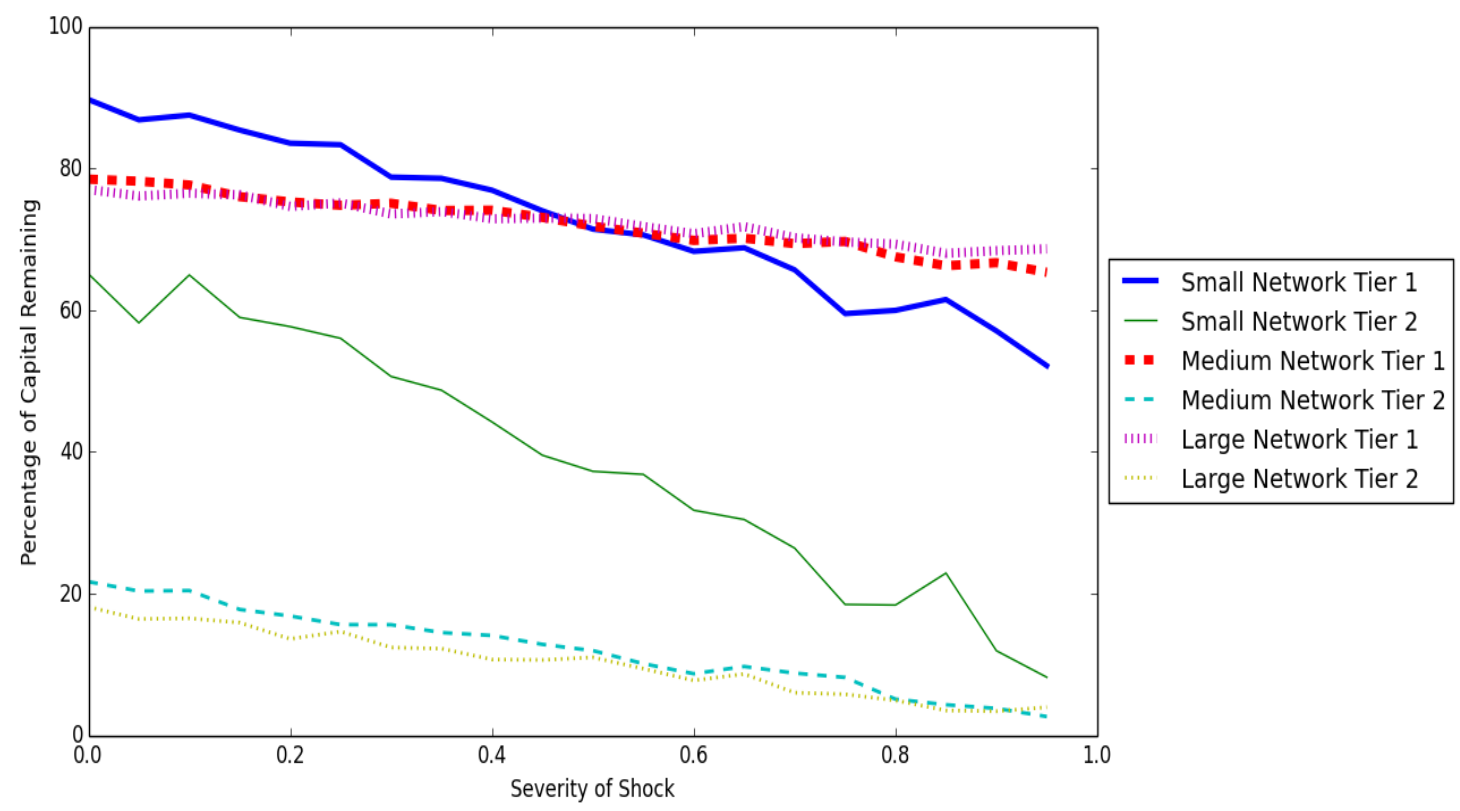

Figure 6: Effect of Size in a Heterogeneous Network

In Figure 5, we plot the residual capital as the severity of the shock is varied. We plot this for three different values of inter-tier connectivity (how many Tier 2 banks are connected to one Tier 1 bank) - 5, 25 and 50 . In general for all the graphs as the severity of the shock increases the residual capital decreases too - which is expected. It is to be noted that at low connectivity (Inter-tier connectivity $=5$ ), as the severity of shock increases the decrement in residual capital for Tier 1 bank is drastic (from $77 \%$ to $38 \%$ ). At low connectivity, if Tier 2 banks fails, due to low connectivity between Tier 1 and Tier 2 , the failure is easily propagated to Tier 1 banks and Tier 1 banks get affected.

However, as the connectivity increases (Inter-tier connectivity $=50$ ), with the increase in severity of shock, the decrement in residual capital for Tier 1 bank is little ( $79 \%$ to $70 \%$ ), whereas the decrement in residual capital for Tier 2 bank is drastic from $20 \%$ to $4 \%$. As the Tier 1 bank holds the majority of the capital in the market, the higher inter-tier connectivity reduces the overall reduction of capital due to shock. Thus for more stability of the system higher inter-tier connectivity between tier 1 and tier 2 is preferred. With higher inter-tier connectivity Tier 1 banks loan to multiple small Tier 2 banks. So if few of the Tier 2 banks fail due to wrong investment in Tier 3 sectors, the impact on Tier 1 will be much less.
Conclusion 5: Higher inter-tier connectivity leads to better stability at Tier 1 (big) banks but lower stability at the Tier 2 (small) banks.

Based on conclusion 5, the central authority should encourage big banks to lend to multiple small banks (not few selective small banks) to increase the stability of the financial loan market.

In Figure 6 we look at the effect of size of the network on its stability in heterogeneous network. We combine different pairs of values for the Tier 1 and Tier 2 banks to get the effect of size of the network to its stability. The different combination of values chosen as (Tier 1, Tier 2) are $[$ Small $=(8,10)$, Medium $=(30,50)$, Large $=(50,80)]$. As can be seen from Figure 6, in case of small network size, the remaining capital decreases drastically from $90 \%$ to $50 \%$ in case of Tier 1 and $65 \%$ to $8 \%$ in case of Tier 2 , as the severity of shock is increased from 0 to 0.95 . With the increased size (medium and large network), the remaining capital decreases, but not that dramatically for both Tier 1 and Tier 2. Also, to note that the reduction of capital remaining with the increase of severity of shock in case of medium and large network are very similar. This indicates that the benefit of increased network size in the stability of the network is limited up to certain size of the network. After which the benefit saturates.

Conclusion 6: Increasing the size of the network in both Tier 1 and Tier 2, in case of heterogeneous network increases the stability of the network. 
However the increase is not linear. The benefit of increased size saturates.

Based on conclusion 6, the central banks should encourage higher number of both big banks and small banks in the system than very few "too big to fail" banks to increase the stability of the financial loan market.

\section{Conclusions}

In this paper we discussed how different parameters of the network effect the stability of network with different structures. The paper first analyzes and shows the effect of certain parameters on a Homogeneous network which give us some results which are of more intuitive nature. Then we introduce a tiered network with endogenous loaning mechanism, where we get few interesting results. (1) How increasing the size and connectivity of the tiered network brings more stability to the upper tier, whereas being not so beneficial to the lower one, in this setting, is one of the major contributions of this work. (2) Also seeing that size of

\section{References}

[1] DasGupta Bhaskar, Kaligounder Lakshmi. "On Global Stability of Financial Networks". August 2012.

[2] Upper, Christian. "Simulation methods to assess the danger of contagion in interbank networks". Journal of Financial Stability, 2011.

[3] Acemoglu, Daron, Asuman Ozdaglar, and Alireza Tahbaz-Salehi. "Systemic risk and stability in financial networks". No. w18727. National Bureau of Economic Research, 2013.

[4] Haldane, Andrew G., and Robert M. May. "Systemic risk in banking ecosystems". Nature 469.7330 (2011)

[5] Nier, Erlend, et al. "Network models and financial stability". Journal of Economic Dynamics and Control 31.6 (2007): 2033-2060.

[6] Boss, Michael, et al." Network topology of the interbank network". Quantitative Finance 4.6 (2004)

[7] Battiston, Stefano, et al. "Debtrank: Too central to fail? financial networks, the fed and systemic risk". Scientific reports 2 (2012).

[8] Cont, Rama, Amal Moussa and Edson Bastos e, Network Structure and systemic risk in banking systems." Systemic Risk in Banking Systems (2010).

[9] Markose, Sheri, Simone Giansante, and Ali Rais Shaghaghi. "Too interconnected to fail' financial network of US CDS network: Topological fragility and systemic risk. ”. Journal of Economic Behavior \& Organization83.3 (2012): 627-646.

[10] Haldane, Andrew G. "Rethinking the financial" network. Springer Fachmedien Wiesbaden, 2013.

[11] Co-Pierre Georg "Basel III and Systemic Risk Regulation - What Way Forward?" Global Financial Networks Working Paper Series 17-2011.

[12] Paul Glasserman and H. Peyton Young. "How likely is Contagion in Financial Networks?". Office of Financial Research Working paper, 2015. the network after a certain threshold does not improve stability is another interesting finding of this study.

The results we observed are also in accordance to Business Insider [19] and Guardian [20], where it was noticed that more than 500 banks crashed and more than 10 trillion dollars were lost during the recession of 2008. Out of these 500 banks may be $10-15$ can be classified as top or mid-tier banks, but the rest tended to be smaller in nature. So it can be assessed that the lower tier banks lost significantly more money than the corresponding top tiered banks. Our other result, that the network size has an effect only till a threshold value also supports and augments the findings of the Bloomberg article "Too Big to Fail"[21].

In future we would like to extend our work by working on an endogenous mechanism which given a network would work towards a state with maximum stability.

[13] AD Langmuir. "Changing concepts of airborne infection of acute contagious diseases: a reconsideration of classic epidemiologic theories”. Annals of the New York Academy of Sciences, 1980.

[14] T JA Jacquez, CP Simon, JS Koopman. "The reproduction number in deterministic models of contagious diseases", Comments Theor. Biol, 1991

[15] GL Kaminsky, CM Reinhart. "On crises, contagion, and confusion". Journal of international Economics, 2000 Elsevier

[16] E Hatfield, JT Cacioppo, RL Rapson.

"Emotional contagion". HCR volume 2, 1993

[17] F Allen, D Gale. "Financial contagion", Journal of political economy, 2000 - JSTOR

[18] RS Burt. "Social contagion and innovation: Cohesion versus structural equivalence", American journal of Sociology, 1987 - JSTOR

[19] John Carney, "America Lost 10.2 Trillion in 2008", Business Insider, last accessed on 6/8/2016

[20] DataBlog, "Failed Banks in the US: Mapping the crisis" The Guardian, last accessed on 6/8/16

[21] Yalman Onaran,"Too Big to Fail: To Block Bailouts, Living Wills and Capital Buffers", Bloomberg, last accessed on $6 / 8 / 2016$

[22] Douglas W. Diamond, Philip H. Dybvig, "Bank Runs, Deposit Insurance and Liquidity", The Journal of Political Economy, 1983.

[23] J Sachs, A Tornell, A Velasco, "The Mexican peso crisis: Sudden death or death foretold?" Journal of international economics, 1996 - Elsevier

[24] R Wade, F Veneroso, "The Asian crisis: the high debt model versus the Wall Street-Treasury-IMF complex", New Left Review, 1999

[25] A Damodaran. “Corporate Finance”.1996. ISBN10: 0470384646

[26] Federal Bank of San Francisco." Dr Econ”. Last accessed on 06/10/2016. 